\title{
Cross-modal re-mapping influences the Simon effect
}

\author{
MARIAELENA TAGLIABUE, MARCO ZORZI, and CARLO UMILTÀ \\ University of Padua, Padua, Italy \\ and Università Vita-Salute San Raffaele, Milan, Italy
}

\begin{abstract}
Tagliabue, Zorzi, Umiltà, and Bassignani (2000) showed that one's practicing of a spatially incompatible task influences performance in a Simon task even when the interval between the two tasks is as long as 1 week. In the present study, three experiments were conducted to investigate whether such an effect could be found in a cross-modal paradigm, whereby stimuli in the two tasks were presented in different modalities. Subjects performed either compatible or incompatible mappings in an acoustic spatial compatibility task and, after an interval of $5 \mathrm{~min}, 24 \mathrm{~h}$, or 7 days, performed a visual Simon task. Results show that the spatially incompatible mapping task affected performance in the Simon task: The Simon effect was absent for all three intervals. This pattern is similar to the results of the Tagliabue et al. study, in which both tasks were performed in the same (visual) modality. Our findings disprove possible explanations based on episodic/contextual effects and support the hypothesis of a long-lasting spatial remapping that is not modality specific.
\end{abstract}

In a typical Simon task, subjects are asked to press one of two keys in response to a nonspatial attribute (e.g., color) of a lateralized stimulus. Even though stimulus position is not relevant for response selection, reaction times (RTs) are faster and responses more accurate when stimulus side and response side correspond (corresponding trials) than when they do not (noncorresponding trials). The RT difference between noncorresponding and corresponding conditions (usually in the range of $20-40 \mathrm{msec}$ when visual stimuli are used) is referred to as the Simon effect (see, e.g., Lu \& Proctor, 1995, for review). A widely accepted explanation of the effect is that in the Simon task, a spatial code is generated for the irrelevant stimulus location attribute, which produces interference at the response selection level (e.g., Lu \& Proctor, 1995; Zorzi \& Umiltà, 1995).

The Simon effect, like other response interference phenomena (e.g., the Stroop effect), is thought to be very robust and relatively impermeable to the effects of practice or strategies. However, the results of two recent studies revealed that performance, prior to the Simon task, under conditions in which the spatial dimension is task relevant, alters the Simon effect in a dramatic way (Proctor \& Lu, 1999; Tagliabue, Zorzi, Umiltà, \& Bassignani, 2000). Proctor and Lu demonstrated that practice with about 900 trials of

This work was supported by a grant from MURST to C.U. and by a McDonnell-Pew Cognitive Neuroscience Program grant to M.Z. and C.U. M.T. was supported by the University of Padua through a research fellowship. The authors thank Elena Giachi and Cristina Olivieri for helping in data collection and B. Hommel and R. Proctor for helpful comments on an earlier version of the paper. Correspondence should be addressed to C. Umilità, Dipartimento di Psicologia Generale, Università di Padova, via Venezia 8,35131 Padova, Italy (e-mail: carlo.umilta@unipd.it).

—Accepted by previous editorial team an incompatible spatial mapping (with stimulus position as the task relevant dimension) led to a reverse Simon effect when stimulus location became subsequently irrelevant. In Tagliabue et al., subjects performed a Simon task after just 72 trials of a spatially compatible or a spatially incompatible task. Results showed a regular Simon effect after the spatially compatible task and a null or reverse Simon effect after the spatially incompatibletask. Even more striking was that the size of the Simon effect after the incompatibletask depended on the interval between the two tasks: There was no Simon effect with delays of 5 min and of $24 \mathrm{~h}$, whereas the effect was reversed after an interval of 7 days.

Tagliabue et al. (2000) interpreted their data on the basis of dual-route models, which, in their various versions (see, e.g., De Jong, Liang, \& Lauber, 1994; Kornblum, Hasbroucq, \& Osman, 1990; Zorzi \& Umiltà, 1995), maintain that the onset of the imperative stimulus activates two parallel pathways. One pathway, referred to as a conditional (or controlled) route, codes the nonspatial, task-relevant stimulus dimension and leads to the selection of the correct response on the basis of task instructions. The other pathway, referred to as an unconditional (or automatic) route, codes the spatial, task-irrelevant stimulus dimension, becoming active irrespective of stimulus identification or task instructions. Barber and O'Leary (1997; see also Umiltà \& Zorzi, 1997) distinguished these two pathways on the basis of their reliance on short-term memory associations (STM links; i.e., the conditional route) or longterm memory associations (LTM links; i.e., the unconditional route).

This approach predicts that the magnitude of the Simon effect should depend on the relative strength of activation of the two routes. When the imperative stimulus appears, its position activates the LTM-based, unconditional route, 
thus priming the corresponding response. At the same time, the STM-based conditional route activates the correct response depending on the nonspatial information. If both routes converge on the same response, RT is fast; if not, the incorrect response must be inhibited, and selection of the correct response requires extra time (see Kornblum, Stevens, Whipple, \& Requin, 1999; Tagliabue et al., 2000; Zorzi \& Umiltà, 1995, for computational models).

Tagliabue et al. (2000) used computer simulations and time-course analyses of the RT data to contrast two different hypotheses regarding how previous practice with a spatially incompatible task might change the cognitive architecture to produce a reverse Simon effect. The first hypothesis was that previous practice produces a modification of LTM links (i.e., the unconditional route); the second hypothesis was that the STM links set up to perform the spatially incompatible task (i.e., a conditional pathway) are still active when the Simon task is later performed. The data support the latter hypothesis. Note that this explanation is somewhat at odds with the notion of STM links, which, by definition, should be brief and transient (i.e., active only for the duration of the task at hand). The finding of a reverse Simon effect 7 days after execution of the spatially incompatible task can be taken as evidence of a consolidation of the putative STM links. Therefore, the distinction between the two types of memory links would seem to find a better grounding within the framework of the hippocampal versus neocortical memory systems (e.g., McClelland, McNaughton, \& O'Reilly, 1995). The hippocampal system allows rapid learning of new (and arbitrary) associations, which can gradually consolidate to become integrated in the neocortical system.

In the present study, we aimed to further investigate the nature of the changes induced by practicing spatially compatible and incompatible tasks. In particular, we asked whether spatial associations that are "carried over" onto the Simon task can be defined as being truly spatial (in the more abstract sense), or whether their nature can be better described as episodic (or contextual). In the latter case, the effect should be limited to conditions in which the stimuli have a substantial degree of similarity across the two tasks (e.g., lateralized visual stimuli in both tasks). Note that the former hypothesis was partly and indirectly supported in Experiment 3 of Tagliabue et al. (2000) by the fact that the spatially incompatible task affected performance in the Simon task even when different stimulus colors were used in the two tasks. Similarly, Proctor and Lu's (1999) Experiment 3 showed that the incompatible location task affected a Simon task with letter stimuli when stimuli in practice trials were color circles.

More direct evidence that the spatial associations are not strictly related to the experimental context could be gathered by employing a cross-modal paradigm, whereby spatial information in the compatibility task and in the Simon task are conveyed by different modalities (e.g., acoustic vs. visual). In other words, evidence that the effects of practicing a spatially incompatible task can transfer across modalities would demonstrate, in a more convincing way, the existence of a true spatial remapping.

The issue of a possible cross-modal transfer of the effects of STM links is particularly relevant in view of the recent study by Proctor, Marble, and Vu (2000). They had their subjects perform in Simon-task trials intermixed with spatially incompatible trials. In different experiments, locational information was provided by the physical location of the stimulus, by a location word, or by direction of an arrow. Results showed that the Simon effect reversed only on condition that the mode of presentation of the locational information was the same when location was task relevant (i.e., spatially incompatible trials) and when location was task irrelevant (i.e., Simon-task trials). In contrast, the Simon effect did not reverse, but rather a regular Simon effect was often found, when mode of locational information presentation differed between location-relevant and location-irrelevant trials. Proctor et al. concluded that STM links between location of the stimulus and location of the response produced by location-relevant trials are mode specific. That might hold when different modes of presentation are used, in which location information is provided verbally or symbolically. However, a different picture is likely to emerge when locational information is always provided by stimulus physical location but in different sensory modalities.

\section{EXPERIMENT 1}

The aim of Experiment 1 was to investigate whether practice of a task in which the spatial dimension is task relevant would affect the subsequent performance on a task in which it is not, when the stimuli in the two conditions are presented in different modalities. Accordingly, the subjects performed a spatial compatibility task (with either compatible or incompatible mappings) in the acoustic modality and were then transferred to a visual Simon task.

\section{Method}

Subjects. Thirty-two students of the University of Padua (aged 19-30) participated in the experiment. They were all right-handed, had normal or corrected-to-normal vision, and were not aware of the purpose of the experiment. They were randomly assigned to two groups of 16 subjects, each group performing a different version of the spatial compatibility task.

Apparatus and Stimuli. The subjects were seated in front of an IBM 386 computer, connected to a NEC Multisync 3FG color monitor through a VGA card. The experiment was run using the Micro Experimental Laboratory (MEL, version 2.0) software system. For the auditory spatial compatibility task, the stimuli were administered through stereo headphones. Sounds were delivered to the left channel, to the right channel, or to both, generating monaural or binaural stimuli. In the visual Simon task, the subjects did not wear headphones. In both tasks, a central $0.7^{\circ} \times 0.7^{\circ}$ cross was presented at fixation. In the auditory spatial compatibility task, a 440-Hz warning signal was presented for $300 \mathrm{msec}$ (via the headphones) at the onset of the cross. No sound was delivered in the Simon task in order to avoid any possible interference due to the acoustic task previously performed. 
Target stimuli were monaural $1800-\mathrm{Hz}$ sounds for the auditory task and two $1^{\circ} \times 1^{\circ}$ colored squares (green or red), shown $10^{\circ}$ to the right or to the left of fixation, for the visual task. Responses were executed by pressing (with the left or the right index finger) one of two keys on the computer keyboard (the "f" or "k" character, covered by a white sticking label, on the left and on the right of the body midline, respectively).

Procedure. There were three experimental tasks: two spatial compatibility tasks (compatible and incompatible mapping, respectively) and the Simon task. Half of the subjects performed the spatially compatible task (Task C), whereas the other half performed the spatially incompatible task (Task I). Then, after Task C or I, both groups performed the Simon task (Task $S$ ) in the visual modality. Between Task $\mathrm{C}$ or I and Task $\mathrm{S}$ there was an interval of approximately $5 \mathrm{~min}$.

Trials began with presentation of the fixation cross that remained visible for $1,800 \mathrm{msec}$. At the offset of fixation, the imperative stimulus appeared for $100 \mathrm{msec}$. After 1,700 $\mathrm{msec}$, a new trial began. The maximum allowed response time was $1,500 \mathrm{msec}$.

In Task C, the subjects were instructed to press the key on the same side as the stimulated ear, whereas in Task I they were to press the key on the opposite side. In Task S, half of the subjects were instructed to press the right key in response to the red square and the left key in response to the green square. The opposite mapping was assigned to the other subjects.

For Task C or I, stimuli were 72 (36 on the left and 36 on the right), and for Task S, they were 144 (equally distributed across the four positions by color conditions). Experimental trials were preceded by as many practice trials as were needed for obtaining 10 correct trials. No subject was given more than 12 practice trials.

\section{Results and Discussion}

Correct RTs (with exclusion of anticipation, i.e., RTs faster than $150 \mathrm{msec}$ ) and errors (including anticipations) were submitted to analyses of variance (ANOVAs) and, when necessary, pairwise comparisons were carried out with the Newman-Keuls method. First, we analyzed data from Task C or I to make sure that the spatial compatibility effect was present, this was necessary for subsequent analyses. The ANOVA had only one between-subjectsfactor, type of spatial task (Task C vs. Task I). RTs were significantly faster for Task C $(387 \mathrm{msec})$ than for Task I $(558 \mathrm{msec})\left[F(1,30)=38.88, M S_{\mathrm{e}}=6,010, p<.001\right]$, with a spatial compatibility effect of $171 \mathrm{msec}$. The same analysis was carried out on error rates. No difference between groups was found: Errors were $1.3 \%$ in Task $\mathrm{C}$ and $2 \%$ in Task I.

For Task S, data were submitted to an ANOVA with one between-subjects factor (type of spatial task: Task C vs. Task I) and one within-subjects factor (type of response: corresponding vs. noncorresponding). The two main effects were significant for type of spatial task $[F(1,30)=$ 8.6, $\left.M S_{\mathrm{e}}=5,849, p<.01\right]$ and for type of response $\left[F(1,30)=16.83, M S_{\mathrm{e}}=433, p<.001\right]$. Furthermore, the interaction between type of spatial task and type of response was significant $\left[F(1,30)=4.91, M S_{\mathrm{e}}=433, p<.05\right]$.

The main effect of type of spatial task showed that the subjects who performed Task C first were faster in Task S than the subjects who performed Task I first (445 vs. $501 \mathrm{msec}$ ). The main effect of response type showed that corresponding responses were faster than noncorresponding responses (463 vs. $484 \mathrm{msec}$ ). Globally, there was a 21msec Simon effect. More interestingly, the interaction between the two factors showed that, in the group who performed Task $\mathrm{C}$ first, corresponding responses were $33 \mathrm{msec}$ faster than noncorresponding responses (429 vs. $462 \mathrm{msec}, p<.001)$, whereas in the Task I group, the Simon effect was small and nonsignificant $(10 \mathrm{msec} ; 496$ vs. 506 msec; see Table 1).

The ANOVA on error data, identical to that performed on RT data, showed a significant effect only of response type $\left[F(1,30)=5.87, M S_{\mathrm{e}}=3.0, p<.05\right]$. Errors were $2.7 \%$ for corresponding responses and $4.2 \%$ for noncorresponding responses.

The results of the present experiment showed that in the Task $\mathrm{C}$ group, the overall Simon effect was $33 \mathrm{msec}$, whereas in the Task I group, it was much smaller and nonsignificant $(10 \mathrm{msec})$. Therefore, practice with a spatially incompatible mapping did affect performance in the Simon task across modalities, similar to what happened in experiments in which both Task C or I and Task S were carried out in the same (visual) modality (Proctor \& Lu, 1999; Tagliabue et al., 2000).

Table 1

Reaction Times (RT, in Milleseconds) and Error Rates (ER) for Each Experiment, Showing a Regular Simon Effect for Task C Groups and a Null Simon Effect for Task I Groups

\begin{tabular}{|c|c|c|c|c|c|c|}
\hline \multirow[b]{3}{*}{ Group } & \multirow[b]{3}{*}{ Time Interval } & \multicolumn{4}{|c|}{ Responses } & \multirow[b]{3}{*}{ Effect Size } \\
\hline & & \multicolumn{2}{|c|}{ Corresponding } & \multicolumn{2}{|c|}{ Noncorresponding } & \\
\hline & & RT & ER & RT & ER & \\
\hline \multicolumn{7}{|c|}{ Experiment 1} \\
\hline $\mathrm{C}$ & $5 \mathrm{~min}$ & 429 & 2.2 & 462 & 4.4 & 33 \\
\hline I & $5 \mathrm{~min}$ & 496 & 3.3 & 506 & 4.1 & 10 \\
\hline \multicolumn{7}{|c|}{ Experiment 2} \\
\hline $\mathrm{C}$ & $24 \mathrm{~h}$ & 469 & 1.6 & 494 & 4.1 & 25 \\
\hline I & $24 \mathrm{~h}$ & 458 & 2.9 & 460 & 3.5 & 2 \\
\hline \multicolumn{7}{|c|}{ Experiment 3} \\
\hline $\mathrm{C}$ & 7 days & 446 & 2.7 & 491 & 5.1 & 45 \\
\hline $\mathrm{I}$ & 7 days & 480 & 3.6 & 484 & 2.8 & 4 \\
\hline
\end{tabular}




\section{EXPERIMENT 2}

The purpose and procedure of Experiment 2 were the same as those of Experiment 1, with the exception that the interval between the spatial compatibility task and the Simon task was $24 \mathrm{~h}$. In fact, Tagliabue et al. (2000) showed that the interval of $24 \mathrm{~h}$ between the incompatible task and the Simon task (both in the visual modality) produced the same effect as the 5-min interval did. Thus, in order to establish that the acoustic incompatibletask induced the same kind of remapping as the incompatible visual task, it was crucial to demonstrate that the influence of the acoustic practice task on the Simon task was long lasting.

\section{Method}

Subjects. Thirty-two subjects, all blind to the purpose of the experiment and selected as before, participated in the experiment. They had not participated in the previous experiment.

Apparatus, Stimuli, and Procedure. These were identical to those of Experiment 1, with the exception that the interval between the spatial compatibility task (Tasks C or I) and the Simon task (Task S) was $24 \mathrm{~h}$.

\section{Results and Discussion}

Correct RTs and errors were submitted to the usual ANOVAs. We first analyzed RTs and errors for Task C and Task I. In the ANOVA on RTs, type of spatial task was significant $\left[F(1,30)=6.21, M S_{\mathrm{e}}=4,292, p<.05\right]$. RTs were $414 \mathrm{msec}$ for Task $\mathrm{C}$ and $472 \mathrm{msec}$ for Task I, with a spatial compatibility effect of $58 \mathrm{msec}$. The same factor was significant in the error ANOVA $[F(1,30)=8.47$, $\left.M S_{\mathrm{e}}=1.0, p<.01\right]$. Error rate was $0.6 \%$ in Task $\mathrm{C}$ and $2.2 \%$ in Task I. The spatial compatibility effect was much smaller in this experiment than in Experiment 1, even though Task C and Task I were identical. The fact that errors were not significantly different in Experiment 1, but were in Experiment 2, might suggest that the large difference in RT effects was due, at least in part, to a difference in speed-accuracy criteria.

In the ANOVA performed on the RT data for Task S, with one between-subjects factor and one within-subjects factor as before, the significant sources of variance were type of response $\left[F(1,30)=9.81, M S_{\mathrm{e}}=286, p<.01\right]$ and the interaction between type of spatial task and type of response $\left[F(1,30)=7.23, M S_{\mathrm{e}}=286, p<.05\right]$. The main effect of response type indicated that, globally, corresponding responses were $13 \mathrm{msec}$ faster than noncorresponding responses (464 vs. $477 \mathrm{msec}$ ). As is shown in Table 1, the interaction between type of spatial task and type of response indicates that, for the Task $\mathrm{C}$ group, corresponding responses were faster than noncorresponding responses (469 vs. $494 \mathrm{msec}, p<.001$ ), whereas for the Task I group, corresponding and noncorresponding responses did not differ (458 vs. $460 \mathrm{msec}$, n.s.).

The Simon task tended to be slower for subjects who practiced Task $\mathrm{C}$ than for those who practiced Task I in Experiment 2 (but the difference was not significant), whereas it was significantly faster (445 vs. $501 \mathrm{msec}$ ) in Experi- ment 1. Perhaps this is due to the different retention intervals, in the sense that practicing Task I needs more effort, which, in turn, produces a decay in performance (i.e., global RT), only when the Simon task is practiced immediately after. This seems confirmed by the results of Experiment 3.

As hypothesized, the Task $\mathrm{C}$ group showed a regular Simon effect of $25 \mathrm{msec}$, whereas the Task I group showed a null Simon effect of $2 \mathrm{msec}$.

In the ANOVA on errors, the main effect of response type was significant $\left[F(1,30)=9.25, M S_{\mathrm{e}}=2.0, p<.01\right]$, and the interaction between type of spatial task and type of response was marginally significant $[F(1,30)=3.99$, $\left.M S_{\mathrm{e}}=2.0, p=.054\right]$. The trend was coherent with that of RTs: Group C subjects made fewer errors on corresponding trials $(1.6 \%)$ than on noncorresponding trials (4.1\%), whereas Group I subjects showed similar error rates in the two conditions ( $2.9 \%$ vs. $3.5 \%$ ).

It can be concluded that practice with spatially incompatible mappings does affect performance in the Simon task by making the Simon effect disappear when the Simon task is performed immediately after (Experiment 1) or 1 day after (Experiment 2) the spatial compatibility task. In other words, practice of the spatially compatible mapping seems not to affect the Simon task.

\section{EXPERIMENT 3}

In Experiment 3, following Tagliabue et al. (2000), we completed the range of intervals between the spatial compatibility task and the Simon task by employing a delay of 7 days. This is the interval that proved to be the most effective in the experiments of Tagliabue et al., in which both tasks were carried out in the visual modality. In their Experiment 4, in which a 5-min interval was used, the Simon effect was absent after Task I (as in Experiments 1 and 2 of the present study). In contrast, the Simon effect was reversed in their Experiment 5, in which a 7-day interval was used. Thus we expected to observe the reversal of the Simon effect, with a delay of 7 days, also when the spatially incompatible task was in the auditory modality.

\section{Method}

Subjects. Thirty-two subjects recruited as before took part in the experiment. None had taken part in the previous experiments.

Apparatus and Stimuli. These were the same as those in Experiment 1.

Procedure. The only difference with respect to Experiment 1 was that the interval between Task $\mathrm{C}$ or I and Task S was 7 days.

\section{Results and Discussion}

The first ANOVA compared RTs in Task C and Task I. The between-subjects factor was significant $[F(1,30)=$ 19.83, $\left.M S_{\mathrm{e}}=5,933, p<.001\right]$ : RTs were $398 \mathrm{msec}$ for Task C and 519 msec for Task I, showing a spatial compatibility effect of $121 \mathrm{msec}$. The ANOVA on errors showed no difference between groups. The subjects made $0.9 \%$ errors in Task $\mathrm{C}$ and $1.6 \%$ in Task I. 
In the ANOVA conducted on the RT data for Task $\mathrm{S}$, as before, the between-subjects factor was type of spatial task, and the within-subjects factor was type of response. Both the main effect of response type and the interaction between type of spatial task and type of response were significant $\left[F(1,30)=17.54, M S_{\mathrm{e}}=556, p<.001 ; F(1,30)=11.79\right.$, $M S_{\mathrm{e}}=556, p<.01$, respectively]. The significance of the within-subjectsfactor shows that there was a global Simon effect of $25 \mathrm{msec}$ : Corresponding responses were faster than noncorresponding responses (463 vs. $488 \mathrm{msec}$ ).

More interestingly, the significance of the interaction between type of spatial task and type of response indicated that the two spatial tasks had different influences on performance in Task S. As can be seen in Table 1, the Task C group showed a regular Simon effect, whereby corresponding responses were $45 \mathrm{msec}$ faster than noncorresponding responses (446 vs. $491 \mathrm{msec}, p<.001$ ). In contrast, the Task I group showed a 4-msec nonsignificant regular Simon effect ( $480 \mathrm{msec}$ for corresponding responses vs. $484 \mathrm{msec}$ for noncorresponding responses).

The same ANOVA on errors showed that only the interaction between type of spatial task and type of response reached significance $\left[F(1,30)=5.77, M S_{\mathrm{e}}=4.0, p<.05\right]$. Similar to the RT data, the subjects who performed Task S after Task $\mathrm{C}$ made fewer errors when stimulus position and response position corresponded than when they did not $(2.7 \%$ vs. $5.1 \%, p=.08)$, whereas the subjects who performed Task S after Task I showed the opposite trend (3.6\% vs. $2.8 \%$, n.s.).

The results of the present experiment suggest that practice with incompatible spatial mapping does not reverse the Simon effect when the spatially incompatible task and the Simon tasks are administered in different modalities, even when the interval between the two tasks is of 1 week. Rather, the results are similar to the two previous experiments that employed intervals of $5 \mathrm{~min}$ and $24 \mathrm{~h}$.

\section{GENERAL DISCUSSION}

The purpose of the present study was to investigate whether performance of the incompatible mapping of a spatial compatibility task administered in the acoustic modality interfered with a Simon task administered in the visual modality and executed after an interval ranging between 5 min and 1 week. This kind of transfer effect has previously been observed (Proctor \& Lu, 1999; Tagliabue et al., 2000) when the spatial compatibility task and the Simon task were performed in the same (visual) modality. In particular, Proctor and $\mathrm{Lu}$ demonstrated that subjects who performed 900 spatially incompatible trials before the Simon task showed a reverse Simon effect. Tagliabue et al. demonstrated that 72 spatially incompatible trials were sufficient to produce the reversal of the Simon effect, when a 1-week delay was interposed between the two tasks. They explained this result, in line with dual-route models (De Jong et al., 1994; Kornblum et al., 1990; Zorzi \& Umiltà, 1995), by assuming that subjects performing
Task I set up STM links to produce correct, spatially incompatible responses. These links, rather than being brief and transient, remain active and can therefore affect performance when the Simon task is later performed. During the time lapse between the two tasks, STM links consolidate and their strength increases over time. Thus, when the Simon task is performed 1 week after the spatially incompatible task, STM links have a stronger influence on the Simon task compared with when the delay between the two tasks is shorter ( 5 min or $24 \mathrm{~h}$ ).

In the present study, the use of different modalities (acoustic vs. visual) in the two tasks was specifically designed to investigate whether the long-lasting effects of STM links are modality specific or not. If they are modality specific, practice of an acoustic incompatible spatial mapping should not have affected performance in a subsequent visual Simon task. In the present experiments, the Simon effect was always present when Task $C$ preceded the Simon task, whereas it was absent for the groups who practiced Task I first. Overall, the results of Experiments 1, 2, and 3 were similar to those of Tagliabue et al. (2000), in which both tasks were carried out in the same (visual) modality. The only notable difference between the two studies concerns the results of Experiment 3 . That is, the Simon effect did not reverse when the Simon task was performed 1 week after Task I. We conducted an ANOVA to compare old data obtained after visual spatial compatibility practice tasks (7-day interval; Experiment 5 of Tagliabue et al., 2000) with the present data from Experiment 3. Modality and type of spatial task were the two between-subjects factors, whereas response type was the sole within-subjects factor. The ANOVA shows that the three-way interaction was not significant $(F<1.0)$. Thus, it might be concluded, for the time being, that studies using different modalities have shown similar patterns of results.

Finally, it is important to note that the present results are $n o t$ at odds with those of Proctor et al. (2000). Proctor et al. showed that locational relevant trials had no influence on locational irrelevant trials when the mode of locational information presentation (physical position, locational word, or arrow direction) changed between location-relevant trials and location-irrelevant trials. Here, we have shown that the influence of locational relevant trials does occur across modalities when locational information is conveyed in either modality by the physical position of the stimulus.

In conclusion, the results of the present cross-modal experiments strongly suggest that the effects of practicing a spatially incompatible task cannot be explained by episodic/contextual factors but must be ascribed to a process of spatial remapping that is not modality specific.

\section{REFERENCES}

BARBER,P., \& O'LEARY,M. (1997). The relevance of salience: Towards an activational account of irrelevant stimulus-response compatibility effects. In B. Hommel \& W. Prinz (Eds.), Theoretical issues in stimulusresponse compatibility (pp. 135-172). Amsterdam: North-Holland. De Jong, R., Liang, C. C., \& Lauber, E. (1994). Conditional and un- 
conditional automaticity: A dual-process model of effects of spatial stimulus-response correspondence. Journal of Experimental Psychology: Human Perception \& Performance, 20, 731-750.

Kornblum, S., Hasbroucq, T., \& Osman, A. (1990). Dimensional overlap: Cognitive basis for stimulus-response compatibility: A model and taxonomy. Psychological Review, 97, 253-270.

Kornblum, S., Stevens, G. T., Whipple, A., \& Requin, J. (1999). The effects of irrelevant stimuli: 1 . The time course of stimulus-stimulus and stimulus-response consistency effects with Stroop-like stimuli, Simon-like tasks, and their factorial combinations. Journal of Experimental Psychology: Human Perception \& Performance, 25, 688-714

Lu, C.-H., \& Proctor, R. W. (1995). The influence of irrelevant location information on performance: A review of the Simon and spatial Stroop effects. Psychonomic Bulletin \& Review, 2, 174-207.

McClelland, J. L., McNaughton, B. L., \& O'Reilly, R. C. (1995). Why there are complementary learning systems in the hippocampus and neocortex: Insights from the successes and failures of connectionist models of learning and memory. Psychological Review, 102, 419-437.

Proctor, R. W., \& Lu, C.-H. (1999). Processing irrelevant location in- formation: Practice and transfer effects in choice-reaction tasks. Memory \& Cognition, 27, 63-77.

Proctor, R. W., Marble, J. G., \& Vu, K. P. L. (2000). Mixing incompatibly mapped location-relevant trials with location-irrelevant trials: Effects of stimulus mode on the reverse Simon effect. Psychological Research, 64, 11-24.

Tagliabue, M., Zorzi, M., Umiltà, C., \& Bassignani, F. (2000). The role of LTM links and STM links in the Simon effect. Journal of Experimental Psychology: Human Perception \& Performance, 26, 648670.

Umiltà, C., \& Zorzi, M. (1997). Commentary on Barber and O'Leary: Learning and attention in S-R compatibility. In B. Hommel \& W. Prinz (Eds.), Theoretical issues in stimulus-response compatibility (pp. 173178). Amsterdam: North-Holland.

ZorZi, M., \& UMiltà, C. (1995). A computational model of the Simon effect. Psychological Research, 58, 193-205.

(Manuscript received June 2, 2000; revision accepted for publication July 10, 2001.) 